\title{
Unidirectional Pumping of Phonons by Magnetization Dynamics
}

\author{
Xiang Zhang $\odot,{ }^{1}$ Gerrit E. W. Bauer $\odot,{ }^{2,1}$ and Tao Yu $\odot^{3,1, *}$ \\ ${ }^{1}$ Kavli Institute of NanoScience, Delft University of Technology, 2628 CJ Delft, Netherlands \\ ${ }^{2}$ WPI-AIMR \& Institute for Materials Research \& CSRN, Tohoku University, Sendai 980-8577, Japan \\ ${ }^{3}$ Max Planck Institute for the Structure and Dynamics of Matter, 22761 Hamburg, Germany
}

(Received 5 March 2020; revised 23 July 2020; accepted 24 July 2020; published 14 August 2020)

\begin{abstract}
We propose a method to control surface phonon transport by weak magnetic fields based on the pumping of surface acoustic waves (SAWs) by magnetostriction. We predict that the magnetization dynamics of a nanowire on top of a dielectric films injects SAWs with opposite angular momenta into opposite directions. Two parallel nanowires form a phononic cavity that at magnetic resonances pump a unidirectional SAW current into half of the substrate.
\end{abstract}

DOI: 10.1103/PhysRevLett.125.077203

Introduction.-Surface acoustic waves (SAWs) on the surface of high-quality piezoelectric crystals are frequently employed for traditional signal processing [1,2], but are also excellent mediators for coherent information exchange between distant quantum systems such as superconducting qubits and/or nitrogen-vacancy centers [3-6]. Piezoelectrically excited coherent SAWs drive the ferromagnetic resonance (FMR) by magnetostriction [7-13], excite spin waves parametrically [14], and generate electron spin currents by the rotation-spin coupling $[15,16]$. Conventional insulators often have good acoustic quality but only small piezoelectric effects, rendering the direct excitation, manipulation, and detection of the coherent SAWs challenging. The phonon pumping [17], i.e., the excitation of bulk sound waves in a high-quality acoustic insulator by the dynamics of a proximity magnetic layer via the magnetoelastic coupling $[18,19]$, may be useful here. Bulk phonons in the insulator gadolinium gallium garnet (GGG) can couple two yttrium iron garnet (YIG) magnetic layers over millimeters [20,21].

Here we address the coherent excitation and manipulation of Rayleigh SAWs by magnetization dynamics, which is possible in a lateral planar configuration with ferromagnetic nanowires on top of a high-quality nonmagnetic insulator, as illustrated in Fig. 1. Similar configurations on magnetic substrates led to the electrical detection of diffuse magnon transport $[22,23]$ and discovery of nonreciprocal magnon propagation [24], i.e., the generation of a unidirectional spin current in half space $[25,26]$. Magnetic

Published by the American Physical Society under the terms of the Creative Commons Attribution 4.0 International license. Further distribution of this work must maintain attribution to the author(s) and the published article's title, journal citation, and DOI. Open access publication funded by the Max Planck Society. stray fields of the magnetization dynamics also generate chiral electron [27] and waveguide photon [28] transport. The unidirectional excitation of SAWs is important for acoustic device applications [29], which conventionally is achieved by metal electrodes on a piezoelectric crystal such that reflected SAWs constructively interfere with the source. This is a pure geometrical effect that is efficient at sub-GHz frequencies and sample dimensions that match the SAW wavelength $[29,30]$.

We focus on the unidirectional [31] excitation of SAWs via magnetic nanostructures on top of a dielectric substrate that are brought into FMR by external microwaves. We predict effects that are very different from the reported nonreciprocity, i.e., a sound velocity that depends on direction [11,32], which is enhanced in magnetic multilayers on top of a piezoelectric substrate [33-35]. The magnetic order of, e.g., a wire on top of a dielectric, does not couple nonreciprocally to the surface phonons in the configuration in Fig. 1, but excites both left- and rightpropagating phonons, even though the angular momentum

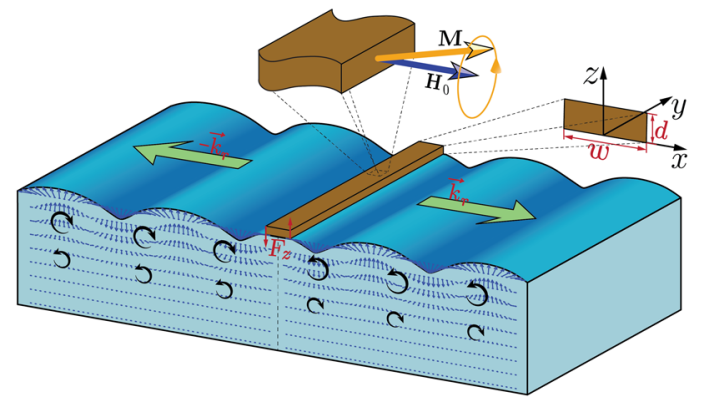

FIG. 1. Surface-phonon pumping by one magnetic nanowire (brown) on top of the acoustic insulator (blue). A static magnetic field $\mathbf{H}_{0}$ applied in the $\vec{x}$ direction saturates the magnetization. The pumped Rayleigh SAWs by the nanowire FMR propagate and rotate in opposite directions at the two sides of the nanowire as indicated by the green and black arrows, respectively. 
current has a direction because of the momentum-rotation coupling of Rayleigh SAWs. However, we predict robust unidirectional excitation of SAW phonons in a phononic cavity formed by two parallel wires. The SAWs actuated by the first wire interact with the second one (which does not see the microwaves) and excite its magnetization, which in turn emits phonons. The phonons from both sources interfere destructively on half of the surface and the net phonon pumping becomes unidirectional. Constructive interference between the two nanowires induces standing SAWs as in a Fabry-Pérot cavity. Conventional unidirectional electric transducer $[29,30]$ operate by a pure geometrical interference effect that works only for a fixed sub-GHz frequency. Our magnetic unidirectional transducer operates by a dynamical phase shift and provides new functionalities, such as robust high frequency tunability and switchability.

Model.-We consider a rectangular magnetic nanowire (YIG) on top of the surface of a thick dielectric (GGG) that spans the $x, y$ plane. It extends along the $y$ direction with $z \in[0, d]$ and $x \in x_{i}+[-w / 2, w / 2]$, as shown in Fig. 1. For an analytical treatment, $d$ is assumed to be much smaller than the skin depth of the SAWs, such that the displacement field in the wire is nearly uniform in the $z$ dependence. The lattice and elastic parameters at the $\mathrm{YIG} \mid \mathrm{GGG}$ interface match well $[17,20,21,36]$ and are assumed equal. A uniform and sufficiently large static magnetic field $\mathbf{H}_{0}$ along $\vec{x}$ saturates the equilibrium magnetization $\mathbf{M}_{0}=M_{s} \vec{x}$ normal to the wire. We can modulate the magnon-phonon coupling simply by rotating $\mathbf{H}_{0}$.

The system Hamiltonian consists of the elastic energy $\hat{H}_{e}$, the magnetoelastic coupling $\hat{H}_{c}$, and the magnetic energy of the Kittel mode [37],

$$
\hat{H}_{m}=\int d \mathbf{r}\left(-M_{x} H_{0}+\frac{1}{2} N_{x x} M_{x}^{2}+\frac{1}{2} N_{z z} M_{z}^{2}\right),
$$

where $\mathbf{M}=\left(M_{x}, M_{y}, M_{z}\right)^{T}$ is the magnetization vector and the demagnetization constants are taken as $N_{x x} \simeq d /(d+w)$ and $N_{z z} \simeq w /(d+w)$ [25]. Although the predicted effects are classical, we use a quantum description for convenience and future applications in quantum phononics [3-6,38]; we can always recover the classical picture by replacing operators by amplitudes. The transverse magnetization is quantized by the Kittel-magnon operator $\hat{\beta}(t)$ with normalized wave function $m_{y, z}[39,40]$, see Supplemental Material [41]:

$$
\hat{\mathbf{M}}_{y, z}=-\sqrt{2 \gamma \hbar M_{s}}\left(m_{y, z} \hat{\beta}(t)+m_{y, z}^{*} \hat{\beta}^{\dagger}(t)\right),
$$

leading to $\hat{H}_{m}=\hbar \omega_{F} \hat{\beta}^{\dagger} \hat{\beta}$ with frequency

$$
\omega_{F}=\mu_{0} \gamma \sqrt{\left(H_{0}-N_{x x} M_{s}\right)\left(H_{0}-N_{x x} M_{s}+N_{z z} M_{s}\right)} .
$$

Here, $-\gamma$ and $\mu_{0}$ are the gyromagnetic ratio and vacuum permeability.

In our configuration, only the Rayleigh SAWs couple efficiently with the magnet which by their surface nature and long decay length are well suited to exchange information with spatially remote magnets (see Supplemental Material [41]). Sufficiently thin and narrow wires do not affect the substrate strongly, so we may treat them perturbatively. The surface eigenmodes of an isotropic elastic half space read [42]

$$
\begin{aligned}
& \psi_{x}=i k \varphi_{k}\left(e^{q z}-\frac{2 q s}{k^{2}+s^{2}} e^{s z}\right) e^{i k x}, \\
& \psi_{z}=q \varphi_{k}\left(e^{q z}-\frac{2 k^{2}}{k^{2}+s^{2}} e^{s z}\right) e^{i k x},
\end{aligned}
$$

where $q=\sqrt{k^{2}-k_{l}^{2}}$ and $s=\sqrt{k^{2}-k_{t}^{2}}$ with $k_{l}=$ $\omega_{k} \sqrt{\rho /(\lambda+2 \mu)}$ and $k_{t}=\omega_{k} \sqrt{\rho / \mu}$ are the wave vectors for longitudinal and transverse bulk waves, respectively. Here, $\rho$ is the material density, $\mu$ and $\lambda$ are the elastic Lamé constants, and $\varphi_{k}$ is a normalization constant [41]. $\omega_{k}=|k| \eta \sqrt{\mu / \rho}=c_{r}|k|$ represents the eigenfrequency of Rayleigh SAWs with velocity $c_{r}$ and $\eta<1$ is the root of the SAW characteristic equation [42] that depends only on the Lamé constants. The relative phase of the displacement field $\left.\operatorname{Arg}\left(\psi_{z} / \psi_{x}\right)\right|_{z=0}= \pm i$ is opposite for right- and leftpropagating waves, which reflects the rotation-momentum locking [42].

The quantized displacement field $\left(\hat{u}_{x}, \hat{u}_{z}\right)$ can be expanded into the eigenmodes $\boldsymbol{\psi}(k)$ and phonon operators $\hat{b}_{k}(t)[38]$

$\hat{\mathbf{u}}(x, z, t)=\sum_{k}\left[\boldsymbol{\psi}(x, z, k) \hat{b}_{k}(t)+\boldsymbol{\psi}^{*}(x, z, k) \hat{b}_{k}^{\dagger}(t)\right]$.

We normalize the mode amplitudes $\boldsymbol{\psi}$ to recover the elastic Hamiltonian for Rayleigh SAWs [41] such that

$$
\hat{H}_{e}=\rho \int d \mathbf{r} \dot{\hat{\boldsymbol{u}}}^{2}(x, z, t)=\sum_{k} \hbar \omega_{k} \hat{b}_{k}^{\dagger} \hat{b}_{k} .
$$

In YIG films the magnetocrystalline anisotropy, which is important in $\mathrm{CoFeB}[12,43]$, is relatively weak $[17,21,36]$ and the (linearized) magnon-phonon coupling energy is dominated by $\hat{H}_{c}^{m}=\left(B_{\perp} / M_{s}\right) \int\left(\hat{M}_{y} \partial_{x} u_{y}+\right.$ $\left.\hat{M}_{z} \partial_{z} u_{x}+\hat{M}_{z} \partial_{x} u_{z}\right) d \boldsymbol{r}$ with magnetoelastic coupling constant $B_{\perp}[37,44]$. The magnetic wire and nonmagnetic substrate are coupled by the dynamics of the surface strain. We require the interaction between a given SAW and the Kittel mode. By the translational symmetry along the nanowire $y$ direction, the displacement field excited by the Kittel magnon does not depend on $y$, and SAWs propagating along $x$ do not contribute to $u_{y}$. 
The magnetoelastic energy contributed by the magnetic wire with length $L$ then becomes

$$
\begin{aligned}
\hat{H}_{c}^{m}= & \frac{B_{\perp} L}{M_{s}} \int_{0}^{d} \hat{M}_{z}\left(\left.u_{z}\right|_{x=w / 2+x_{i}}-\left.u_{z}\right|_{x=-w / 2+x_{i}}\right) d z \\
& +\frac{B_{\perp} L}{M_{s}} \int_{-w / 2+x_{i}}^{w / 2+x_{i}} \hat{M}_{z}\left(\left.u_{x}\right|_{z=d}-\left.u_{x}\right|_{z=0}\right) d x .
\end{aligned}
$$

We limit attention to the realistic situation in which the wire thickness $d$ is much smaller than the decay length of the SAWs into the bulk. The strain in the magnet then mirrors that of the SAW at $z=0$ of the dielectric and the second term in Eq. (6) vanishes [12],

$$
\hat{H}_{c}^{m} \rightarrow \frac{B_{\perp} L d}{M_{s}} \hat{M}_{z}\left(\left.u_{z}\right|_{x=w / 2+x_{i}}-\left.u_{z}\right|_{x=-w / 2+x_{i}}\right),
$$

that corresponds to an oscillating surface force $\left.\boldsymbol{F}\right|_{x= \pm w / 2+x_{i}}=$ $\mp B_{\perp} L d \hat{M}_{z} / M_{s} \vec{z}$ in the $z$ direction that excites SAWs traveling outwards in both directions [41]. Substituting Eqs. (2) and (4) into Eq. (7), we arrive at the interaction Hamiltonian

$$
\hat{H}_{c}=\hbar \sum_{k} g_{k} \hat{\beta}^{\dagger} \hat{b}_{k}+\text { H.c. }
$$

in which the coupling constant $(q d \ll 1, s d \ll 1)$

$$
g_{k} \simeq-B_{\perp} \sqrt{\frac{\gamma}{M_{s} \rho c_{r}}} \sqrt{\frac{d}{w}} \sin \left(\frac{k w}{2}\right) \xi_{M} \xi_{P} e^{i k x_{i}},
$$

with factors $\xi_{M}$ and $\xi_{P}$ governed by the magnetic and acoustic material parameters [41]. The form factor oscillates and decreases algebraically as a function of nanowire width and phonon wavelength and vanishes when $d, w \rightarrow 0$. The coupling is reciprocal since $\left|g_{k}\right|=\left|g_{-k}\right|$.

$S A W$ pumping.-We now calculate the phonon pumping by a single magnetic nanowire transducer centered at $x_{0}$ and excited by microwave photons represented by the (annihilation) operator $\hat{p}_{\text {in }}$. The Hamiltonian $\hat{H}=\hat{H}_{m}+\hat{H}_{e}+\hat{H}_{c}$ leads to the Heisenberg equation of motion $[45,46]$

$$
\begin{aligned}
d \hat{\beta} / d t & =-i \omega_{F} \hat{\beta}-i \sum_{k}\left|g_{k}\right| e^{i k x_{0}} \hat{b}_{k}-\left(\kappa_{m}+\kappa_{\omega}\right) \hat{\beta} / 2-\sqrt{\kappa_{\omega}} \hat{p}_{\text {in }}, \\
d \hat{b}_{k} / d t & =-i \omega_{k} \hat{b}_{k}-i\left|g_{k}\right| e^{-i k x_{0}} \hat{\beta}-\delta_{k} \hat{b}_{k} / 2
\end{aligned}
$$

where $\kappa_{m}$ and $\delta_{k}$ are the intrinsic damping rates for the nanowire magnon and surface phonon, while $\kappa_{\omega}$ is the radiative damping induced by the microwave field. In the frequency domain, $\hat{\mathcal{O}}(\omega)=\int d t \hat{\mathcal{O}}(t) e^{i \omega t}$, $\hat{\beta}(\omega)=\frac{-i \sqrt{\kappa_{\omega}} \hat{p}_{\text {in }}(\omega)}{\omega-\omega_{F}+i\left(\kappa_{m}+\kappa_{\omega}\right) / 2-\sum_{k}\left|g_{k}\right|^{2} G_{k}(\omega)}$,

$\hat{b}_{k}(\omega)=G_{k}(\omega)\left|g_{k}\right| e^{-i k x_{0}} \hat{\beta}(\omega)$,

where $G_{k}(\omega)=1 /\left(\omega-\omega_{k}+i \delta_{k} / 2\right)$ is the phonon Green function. The additional magnetic damping by the phonon pumping at the FMR $[17,47]$ is given by the imaginary part of the magnon self-energy

$$
\sigma_{k}(\omega)=-\operatorname{Im}\left(\sum_{k}\left|g_{k}\right|^{2} G_{k}(\omega)\right)=\frac{\left|g_{k_{r}}\right|^{2}}{c_{r}},
$$

where we use the on-shell approximation [48,49] with $\omega \rightarrow \omega_{F}$ and $k_{r}=\omega_{F} / c_{r}$. The real part of the self-energy causes a small frequency shift that is absorbed into $\omega_{F}$ in the following.

The displacement field given by Eq. (4) is a superposition of coherent phonons $\left\langle\hat{b}_{k}\right\rangle$ that are excited by the microwave input $\left\langle\hat{p}_{\text {in }}(\omega)\right\rangle$. At resonance $\omega \rightarrow \omega_{F}$, the contour of the $k$ integral must be closed in the upper (lower) half of the complex plane for $x>x_{0}\left(x<x_{0}\right)$, selecting the poles $k_{r}+i \epsilon\left(-k_{r}-i \epsilon\right)$ in the denominator, where $\epsilon$ is the inverse of the phonon propagation length. The low ultrasonic attenuation in GGG at room temperature corresponds to characteristic SAW decay lengths of up to $6 \mathrm{~mm}$ [50]. We can therefore safely disregard the phonon damping $\left(\epsilon \rightarrow 0_{+}\right)$, which leads to displacement fields

$$
\mathbf{u}(z, t)=-\frac{2}{c_{r}} \operatorname{Re}\left\{\begin{array}{ll}
i \boldsymbol{\psi}\left(k_{r}, z\right) g_{k_{r}}^{*}\langle\hat{\beta}(t)\rangle, & x>x_{0} \\
i \boldsymbol{\psi}\left(-k_{r}, z\right) g_{-k_{r}}^{*}\langle\hat{\beta}(t)\rangle, & x<x_{0}
\end{array} .\right.
$$

On the right (left) side of the nanowire $x>x_{0}\left(x<x_{0}\right)$, the right- and left-propagating waves with opposite rotations, whose directions depend on $z$, are pumped as illustrated in Fig. 1. A classical treatment leads to the same result [41].

These phonons carry a constant mechanical angular momentum density $\boldsymbol{l}_{\mathrm{DC}}(x, z)=\rho\langle\mathbf{u} \times \dot{\mathbf{u}}\rangle_{t}$, where the subscript $t$ indicates time average, which is often referred to as phonon spin [18,51,52]:

$$
\begin{aligned}
\boldsymbol{l}_{\mathrm{DC}}(z)= & \left(4 \rho \omega_{F} / c_{r}^{2}\right)|\langle\hat{\beta}\rangle|^{2}\left|g_{k_{r}}\right|^{2} \vec{y} \\
& \times \operatorname{Im}\left\{\begin{array}{ll}
\psi_{x}\left(k_{r}, z\right) \psi_{z}^{*}\left(k_{r}, z\right), & x>x_{0} \\
\psi_{x}\left(-k_{r}, z\right) \psi_{z}^{*}\left(-k_{r}, z\right), & x<x_{0}
\end{array} .\right.
\end{aligned}
$$

$l_{\mathrm{DC}}$ is proportional to the excited magnon population, parallel to the wire, and opposite on both sides of the nanowire since $\psi_{x}(-k) \psi_{z}^{*}(-k)=-\psi_{x}(k) \psi_{z}^{*}(k)$. Into the substrate ( $z$ direction), the SAW eigenmodes have a node at which $l_{\mathrm{DC}}$ changes sign [41] as sketched in Fig. 1. The phonon pumping does not remove angular momentum from the ferromagnet, since only the $x$ component of the magnetic precession is damped. The force on the interface is a superposition of opposite angular momenta 

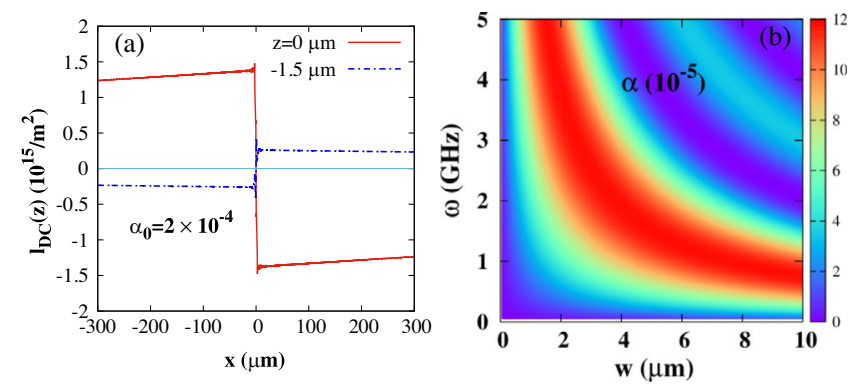

FIG. 2. Pumped phonon spin density [(a)] and additional magnon damping coefficient $\alpha$ [(b)] for a YIG wire on a GGG substrate as a function of FMR frequencies and wire widths.

$2 \mathbf{z}=(\mathbf{z}+i \mathbf{x})+(\mathbf{z}-i \mathbf{x})$ that by the spin-momentum locking couple to phonons moving in opposite directions.

The efficiency of phonon spin pumping depends on the nanowire and substrate. For GGG at room temperature, $\rho=7080 \mathrm{~kg} / \mathrm{m}^{3}, \quad c_{l}=6545 \mathrm{~m} / \mathrm{s}$, and $c_{t}=3531 \mathrm{~m} / \mathrm{s}$ [53], leading to [42] $\eta=0.927, c_{r}=\eta c_{t}=3271.8 \mathrm{~m} / \mathrm{s}$, and $\xi_{P}=0.537$. For YIG [54], $\gamma=1.82 \times 10^{11} \mathrm{~s}^{-1} \mathrm{~T}^{-1}$, $\mu_{0} M_{s}=0.177 \mathrm{~T}$ [55], $B_{\perp}=6.96 \times 10^{5} \mathrm{~J} / \mathrm{m}^{3}$ [17], and $\xi_{M} \approx 1$ when $H_{0}$ is comparable to $M_{s}$. We plot the pumped phonon spin density at different $z$ in Fig. 2(a) with $\omega_{F}=3 \mathrm{GHz}, d=200 \mathrm{~nm}$ and $w=2.5 \mu \mathrm{m}$. We use a small precession cone angle $10^{-3}$ degrees and phonon diffusion length $\sim 6 \mathrm{~mm}$. The spin density is opposite at the two sides of the nanowire and changes sign at larger $z$. Figure 2(b) is a plot of the additional magnon damping coefficient $\alpha=\sigma_{k} / \omega_{F}$ in the dependence of FMR frequency $\omega_{F}$ and nanowire width $w$. We observe geometric resonances $\sim 1 / w$ with $\alpha \lesssim 1 \times 10^{-4}$, which is of the order of the intrinsic Gilbert damping of YIG single crystals $\alpha_{0} \sim 4 \times 10^{-5}$ [56] and films $8 \times 10^{-5}$ [57]. In the thin YIG film, the additional damping $\alpha \sim d$.

Unidirectional phonon pumping.-The single wire emits spin-momentum locked SAWs into two directions. We propose a truly unidirectional phonon source in the form of two parallel and identical nanowires located at $\mathbf{r}_{1}=R_{1} \vec{x}$ and $\mathbf{r}_{2}=R_{2} \vec{x}$, of which only the left one is addressed by a local microwave stripline [58]. The excited phonons below propagate to and are absorbed by the second nanowire. Its dynamics reemits phonons that subsequently interfere with the original ones [20]. Denoting the magnon operators in the left and right nanowires as $\hat{\beta}_{L}$ and $\hat{\beta}_{R}[41]$,

$\hat{\beta}_{R}(\omega)=\frac{\sum_{k}\left|g_{k}\right|^{2} G_{k}(\omega) e^{i k\left(R_{2}-R_{1}\right)}}{\omega-\omega_{F}+i \kappa_{m} / 2-\sum_{k}\left|g_{k}\right|^{2} G_{k}(\omega)} \hat{\beta}_{L}(\omega)$,

$\hat{b}_{k}(\omega)=\left|g_{k}\right| G_{k}(\omega)\left(e^{-i k R_{1}} \hat{\beta}_{L}(\omega)+e^{-i k R_{2}} \hat{\beta}_{R}(\omega)\right)$.

At the FMR $\omega \rightarrow \omega_{F}$,

$$
\hat{\beta}_{R}\left(\omega_{F}\right)=\chi\left(k_{r}\right) e^{i \pi+i k_{r}\left(R_{2}-R_{1}\right)} \hat{\beta}_{L}\left(\omega_{F}\right),
$$

where $\chi\left(k_{r}\right)=\sigma\left(k_{r}\right) /\left[\kappa_{m} / 2+\sigma\left(k_{r}\right)\right]$ modulates the magnetization amplitude in the second wire and $k_{r}\left(R_{2}-R_{1}\right)$ is the phase delay by the phonon transmission. The phase shift $\pi$ reflects the dynamical phase relation between magnons and phonons that is the key for the unidirectionality. This relation can be observed inductively in microwave transmission spectra [41].

By substituting Eq. (16) into (15) at the FMR:

$$
\begin{aligned}
\hat{b}_{-k_{r}} & =\left|g_{k_{r}}\right| G_{k_{r}} e^{i k_{r} R_{1}} \hat{\beta}_{L}\left(\omega_{F}\right)\left[1-\chi\left(k_{r}\right) e^{2 i k_{r}\left(R_{2}-R_{1}\right)}\right], \\
\hat{b}_{k_{r}} & =\left|g_{k_{r}}\right| G_{k_{r}} e^{-i k_{r} R_{1}} \hat{\beta}_{L}\left(\omega_{F}\right)\left[1-\chi\left(k_{r}\right)\right] .
\end{aligned}
$$

In the strong magnon-phonon coupling limit $\sigma\left(k_{r}\right) \gg$ $\kappa_{m} / 2, \chi\left(k_{r}\right) \rightarrow 1$, thus the right-going phonon $k_{r}>0$ is not excited by the double-wire configuration. Finite $\left\langle\hat{b}_{-k_{r}}\right\rangle$ but vanishing $\left\langle\hat{b}_{k_{r}}\right\rangle$ implies a unidirectional phonon current. Such unidirectionality vanishes when the second wire is weakly coupled to the SAW, i.e., $\sigma\left(k_{r}\right) \ll \kappa_{m} / 2$, i.e., phonons transmit without interacting with the magnet.

By Eqs. (4) and (15), the displacement fields of frequency $\omega_{F}$ read

$$
\mathbf{u}(x, t)=\frac{2\left|g_{k_{r}}\right|}{c_{r}} \operatorname{Im} \begin{cases}\boldsymbol{\psi}\left(-k_{r}\right) e^{i k_{r} R_{1}}\left\langle\hat{\beta}_{L}(t)\right\rangle\left(1-\chi\left(k_{r}\right) e^{2 i k_{r}\left(R_{2}-R_{1}\right)}\right) & x<R_{1} \\ e^{i k_{r}\left(R_{2}-R_{1}\right)}\left\langle\hat{\beta}_{L}(t)\right\rangle\left(\boldsymbol{\psi}\left(k_{r}\right) e^{-i k_{r} R_{2}}-\chi\left(k_{r}\right) \boldsymbol{\psi}\left(-k_{r}\right) e^{i k_{r} R_{2}}\right) & \text { for } R_{1}<x<R_{2} \\ \boldsymbol{\psi}\left(k_{r}\right) e^{-i k_{r} R_{1}}\left\langle\hat{\beta}_{L}(t)\right\rangle\left(1-\chi\left(k_{r}\right)\right) & x>R_{2}\end{cases}
$$

When $\chi\left(k_{r}\right) \rightarrow 1$, the displacement field vanishes in the region $x>R_{2}$, but is a traveling wave for $x<R_{1}$. Between the two nanowires with $R_{1}<x<R_{2}$, the SAWs form standing waves with $u_{z} \sim \sin k_{r}\left(x-R_{2}\right)$ and $u_{x} \sim \cos k_{r}\left(x-R_{2}\right)$. The pumping is unidirectional apart from special cases: with frequency or distance $k_{r}\left(R_{2}-R_{1}\right)=n \pi$ with $n \in \mathbb{Z}_{0}$, the SAWs on the left-hand side vanishes as well and the phonon is fully trapped between the two wires to form a cavity. The phonon emission is not perfectly unidirectional when $\chi\left(k_{r}\right)<1$, however. Figure 3(a) is a plot of the magnitude of the displacement field at the GGG surface $|\mathbf{u}(x, z=0)|=$ $\left.\sqrt{u_{x}^{2}+u_{z}^{2}}\right|_{z=0}$ with an intrinsic Gilbert damping $\alpha_{0}=$ $8 \times 10^{-5}$ [57]. We choose the YIG wires of $d=200 \mathrm{~nm}$ and $w=2.5 \mu \mathrm{m}$ to center at $R_{1}=0$ and $R_{2}=30 \mu \mathrm{m}$, and 

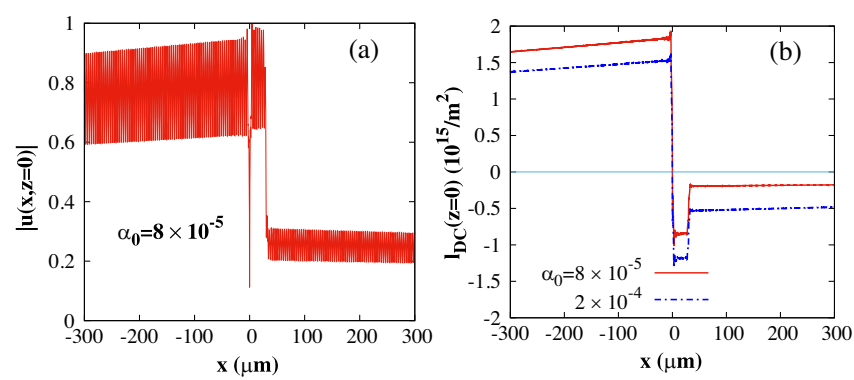

FIG. 3. Snapshot of the displacement field at the GGG surface $|\mathbf{u}|[(\mathrm{a})]$ and phonon spin density [(b)], pumped by a YIG wire at the origin under FMR and modulated by a second YIG wire at $30 \mu \mathrm{m}$.

the Kittel frequency $\omega_{F}=3 \mathrm{GHz}$ such that the additional damping coefficient is $1.2 \times 10^{-4}$. In Fig. 3(b) we plot the phonon (DC) spin density at the GGG surface for a precession cone angle $10^{-3}$ degrees in the left wire. The asymmetry of the pumped phonon spin at the two sides of YIG cavity is not perfect, but clearly survives a larger damping. In YIG|GGG systems, phonon pumping should be measurable even in less than perfect samples. Devices with more than two wires or made from magnetic materials with larger magnetoelasticity may achieve full unidirectionality.

The pumped phonon (spin) can propagate coherently over millimeters on the substrate surface, which is very promising for classical and quantum transport of spin information. It can be measured by Brillouin light scattering [18], the spin-rotation coupling by fabricating a conductor on top of the acoustic medium [15,16], and other techniques [52]. The generation of unidirectionality by interference does not require a nonreciprocal coupling mechanism [25-28] but only an out-of-phase relation of the two fields at resonance. The phenomenon should be universal for many field propagation phenomena, such as exchange coupled magnetic nanowires and films [25] and reciprocally coupled magnons and waveguide photons [28].

Discussion.-In conclusion, we developed a theory for pumping SAWs and proposed a phonon cavity device that realizes unidirectional phonon current in a reciprocal system. When exciting a single magnetic nanowire by microwaves, we predict a passive wire induces the unidirectional phonon current and formation of standing waves in the region between two magnetic nanowires. This mechanism should also lead to a unidirectional spin Seebeck effect [26] generated by a temperature gradient between the magnetic and acoustic insulators [59]. Unidirectionality emerges here from a dynamical phase shift, rather than the purely geometrical interference employed by electrical unidirectional SAW generators $[29,30]$. In the strong coupling regime [20] two magnetic wires on a simple dielectric form a fully unidirectional SAW phonon source or transducer that opens intriguing perspectives for magnonics and spintronics, but also plasmonics [60,61], nano-optics [62], and quantum communication.

This work is financially supported by the Nederlandse Organisatie voor Wetenschappelijk Onderzoek (NWO) as well as JSPS KAKENHI Grant No. 19H006450.

*tao.yu@mpsd.mpg.de

[1] E. A. Ash, A. A. Oliner, G. W. Farnell, H. M. Gerard, A. J. Slobodnik, and H. I. Smith, in Acoustic Surface Waves (Topics in Applied Physics) (Springer, Berlin, 2014).

[2] G. S. Kino, Acoustic Waves: Devices, Imaging, and Analog Signal Processing (Prentice-Hall, New Jersey, 1987).

[3] M. V. Gustafsson, T. Aref, A. F. Kockum, M. K. Ekstrom, G. Johansson, and P. Delsing, Science 346, 207 (2014).

[4] R. Manenti, A. F. Kockum, A. Patterson, T. Behrle, J. Rahamim, G. Tancredi, F. Nori, and P. J. Leek, Nat. Commun. 8, 975 (2017).

[5] K. J. Satzinger, Y. P. Zhong, H.-S. Chang, G. A. Peairs, A. Bienfait, M.-H. Chou et al., Nature (London) 563, 661 (2018).

[6] D. A. Golter, T. Oo, M. Amezcua, I. Lekavicius, K. A. Stewart, and H. Wang, Phys. Rev. X 6, 041060 (2016).

[7] P. Delsing, A. N. Cleland, M. J. Schuetz, J. Knörzer, G. Giedke, J. I. Cirac et al., J. Phys. D 52, 353001 (2019).

[8] Y. Yahagi, B. Harteneck, S. Cabrini, and H. Schmidt, Phys. Rev. B 90, 140405(R) (2014).

[9] M. Weiler, L. Dreher, C. Heeg, H. Huebl, R. Gross, M. S. Brandt, and S. T. B. Goennenwein, Phys. Rev. Lett. 106, 117601 (2011).

[10] L. Dreher, M. Weiler, M. Pernpeintner, H. Huebl, R. Gross, M. S. Brandt, and S. T. B. Goennenwein, Phys. Rev. B 86, 134415 (2012).

[11] R. Sasaki, Y. Nii, Y. Iguchi, and Y. Onose, Phys. Rev. B 95, 020407(R) (2017).

[12] J. Puebla, M. Xu, B. Rana, K. Yamamoto, S. Maekawa, and Y. Otani, J. Phys. D 53, 264002 (2020).

[13] M. Weiler, H. Huebl, F. S. Goerg, F. D. Czeschka, R. Gross, and S. T. B. Goennenwein, Phys. Rev. Lett. 108, 176601 (2012).

[14] I. Lisenkov, A. Jander, and P. Dhagat, Phys. Rev. B 99, 184433 (2019).

[15] M. Matsuo, J. Ieda, K. Harii, E. Saitoh, and S. Maekawa, Phys. Rev. B 87, 180402(R) (2013).

[16] D. Kobayashi, T. Yoshikawa, M. Matsuo, R. Iguchi, S. Maekawa, E. Saitoh, and Y. Nozaki, Phys. Rev. Lett. 119, 077202 (2017).

[17] S. Streib, H. Keshtgar, and G. E. W. Bauer, Phys. Rev. Lett. 121, 027202 (2018).

[18] J. Holanda, D. S. Maior, A. Azevedo, and S. M. Rezende, Nat. Phys. 14, 500 (2018).

[19] C. Berk, M. Jaris, W. Yang, S. Dhuey, S. Cabrini, and H. Schmidt, Nat. Commun. 10, 2652 (2019).

[20] K. An, A. N. Litvinenko, R. Kohno, A. A. Fuad, V. V. Naletov, L. Vila, U. Ebels, G. de Loubens, H. Hurdequint, N. Beaulieu, J. Ben Youssef, N. Vukadinovic, G. E. W. Bauer, A. N. Slavin, V. S. Tiberkevich, and O. Klein, Phys. Rev. B 101, 060407(R) (2020). 
[21] A. Rückriegel and R. A. Duine, Phys. Rev. Lett. 124, 117201 (2020).

[22] K. S. Das, J. Liu, B. J. van Wees, and I. J. Vera-Marun, Nano Lett. 18, 5633 (2018).

[23] L. J. Cornelissen, J. Liu, R. A. Duine, J. Ben Youssef, and B. J. van Wees, Nat. Phys. 11, 1022 (2015).

[24] J. Chen, C. Liu, T. Liu, Y. Xiao, K. Xia, G. E. W. Bauer, M. Wu, and H. Yu, Phys. Rev. Lett. 120, 217202 (2018).

[25] T. Yu, C. P. Liu, H. M. Yu, Y.M. Blanter, and G. E. W. Bauer, Phys. Rev. B 99, 134424 (2019); J. L. Chen, T. Yu, C. Liu, T. Liu, M. Madami, K. Shen, J. Zhang, S. Tu, M. S. Alam, K. Xia, M. Wu, G. Gubbiotti, Y. M. Blanter, G. E. W. Bauer, and H. Yu, Phys. Rev. B 100, 104427 (2019).

[26] T. Yu, Y. M. Blanter, and Gerrit E. W. Bauer, Phys. Rev. Lett. 123, 247202 (2019).

[27] T. Yu and G. E. W. Bauer, Phys. Rev. Lett. 124, 236801 (2020).

[28] T. Yu, Y.-X. Zhang, S. Sharma, X. Zhang, Y. M. Blanter, and G. E. W. Bauer, Phys. Rev. Lett. 124, 107202 (2020).

[29] E. Dieulesaint and D. Royer, Elastic Waves in Solids II: Generation, Acousto-Optic Interaction, Applications (Springer, New York, 2000).

[30] P. Ventura, M. Solal, P. Dufilie, J. M. Hode, and F. Roux, Proc. IEEE Ultrasonic Symp. 1, 1 (1994).

[31] In the literature different terms are used to emphasize different aspects of the same thing. "Unidirectional" refers to the current direction, "chirality" is a fundamental symmetry property that governs this direction, while "nonreciprocity" is an asymmetry of mutual interactions in coherent systems by broken symmetries, of which unidirectionality is an extreme consequence.

[32] R. E. Camley, Surf. Sci. Rep. 7, 103 (1987).

[33] R. Verba, I. Lisenkov, I. Krivorotov, V. Tiberkevich, and A. Slavin, Phys. Rev. Applied 9, 064014 (2018).

[34] R. Verba, V. Tiberkevich, and A. Slavin, Phys. Rev. Applied 12, 054061 (2019).

[35] M. R. Xu, K. Yamamoto, J. Puebla, K. Baumgaertl, B. Rana, K. Miura, H. Takahashi, D. Grundler, S. Maekawa, and Y. Otani, arXiv:2001.05135.

[36] L. D. Landau and E. M. Lifshitz, Theory of Elasticity (Pergamon, Oxford, 1970).

[37] L. D. Landau and E. M. Lifshitz, Electrodynamics of Continuous Media, 2nd ed. (Butterworth-Heinenann, Oxford, 1984).

[38] M. J. A. Schuetz, E. M. Kessler, G. Giedke, L. M. K. Vandersypen, M. D. Lukin, and J. I. Cirac, Phys. Rev. X 5, 031031 (2015).

[39] T. Yu, S. Sharma, Y. M. Blanter, and G. E. W. Bauer, Phys. Rev. B 99, 174402 (2019).
[40] L. R. Walker, Phys. Rev. 105, 390 (1957).

[41] See Supplemental Material at http://link.aps.org/supplemental/ 10.1103/PhysRevLett.125.077203 for the derivation of the magnon and phonon wave function, classical description of SAW pumping, and microwave transmission.

[42] I. A. Viktorov, Rayleigh and Lamb Waves: Physical Theory and Applications (Plenum Press, New York, 1967).

[43] R. Sasaki, Y. Nii, Y. Iguchi, and Y. Onose, Phys. Rev. B 95, 020407(R) (2017).

[44] A. Rückriegel, P. Kopietz, D. A. Bozhko, A. A. Serga, and B. Hillebrands, Phys. Rev. B 89, 184413 (2014).

[45] C. W. Gardiner and M. J. Collett, Phys. Rev. A 31, 3761 (1985).

[46] A. A. Clerk, M. H. Devoret, S. M. Girvin, F. Marquardt, and R. J. Schoelkopf, Rev. Mod. Phys. 82, 1155 (2010).

[47] S. Streib, N. Vidal-Silva, K. Shen, and G. E. W. Bauer, Phys. Rev. B 99, 184442 (2019).

[48] G. D. Mahan, Many Particle Physics (Plenum, New York, 1990).

[49] G. F. Giuliani and G. Vignale, Quantum Theory of the Electron Liquid (Cambridge University Press, Cambridge, England, 2005).

[50] M. Dutoit, J. Appl. Phys. 45, 2836 (1974).

[51] Y. Long, J. Ren, and H. Chen, Proc. Natl. Acad. Sci. U.S.A. 115, 9951 (2018).

[52] D. M. Juraschek and N. A. Spaldin, Phys. Rev. Mater. 3, 064405 (2019).

[53] M. Schreier, A. Kamra, M. Weiler, J. Xiao, G. E. W. Bauer, R. Gross, and S.T.B. Goennenwein, Phys. Rev. B 88, 094410 (2013).

[54] Q. Wang, B. Heinz, R. Verba, M. Kewenig, P. Pirro, M. Schneider, T. Meyer, B. Lägel, C. Dubs, T. Brächer, and A. V. Chumak, Phys. Rev. Lett. 122, 247202 (2019).

[55] A. A. Serga, A. V. Chumak, and B. Hillebrands, J. Phys. D 43, 264002 (2010).

[56] B. M. Yao, T. Yu, Y. S. Gui, J. W. Rao, Y. T. Zhao, W. Lu, and C.-M. Hu, Commun. Phys. 2, 161 (2019).

[57] H. Chang, P. Li, W. Zhang, T. Liu, A. Hoffmann, L. Deng, and M. Wu, IEEE Magn. Lett. 5, 6700104 (2014).

[58] H. Yu, G. Duerr, R. Huber, M. Bahr, T. Schwarze, F. Brandl, and D. Grundler, Nat. Commun. 4, 2702 (2013).

[59] G. E. W. Bauer, E. Saitoh, and B. J. van Wees, Nat. Mat. 11, 391 (2012).

[60] K. Y. Bliokh, D. Smirnova, and F. Nori, Science 348, 1448 (2015).

[61] K. Y. Bliokh and F. Nori, Phys. Rep. 592, 1 (2015).

[62] L. Novotny and B. Hecht, Principles of Nano-Optics (Cambridge University Press, Cambridge, England, 2006). 